\title{
Editorial
}

\section{One Door Opens, One Door Closes -Yielding Path to Bradykinin-Induced Pain}

Howard S. Smith, MD

$t$ has been appreciated for some time that bradykinin (BK) is a significant endogenous mediator of inflammation (1) as well as a potent nociceptive mediator which may contribute to pain $(2,3)$. Bradykinin is a 9-amino cid peptide which is clipped by the serine protease kallikrein from a 110-kDa precursor plasma $\alpha$-globulin known as kininogen. Tissue insult leads to the conversion of 4 an inactive precursor, prekallikrein, to kallikrein, a process which is accelerated by clotting factor XII (Hageman factor) in the presence of negative charges (present on enapthelial cell surfaces) (4). BK tends to act at sites where it is locally formed, as it is rapidly inactivated in the plasma and lungs by peptidases.

The hypersensitivity induced by $B K$ is believed to occur via $B_{2} R$ stimulation with activation of the $G$ protein $\mathrm{Gq}$, the $\alpha$-subunit of which activates phospholipase $\mathrm{C}$ in the cell membrane. PLC $\mathrm{C}_{\beta}$ cleaves phosphatidylinositol 4,5 bisphosphate $\left(\mathrm{PIP}_{2}\right)$ into inositol triphosphate $\left(\mathrm{IP}_{3}\right)$, which contributes to $\mathrm{BK}$-induced acute pain, and diacylglycerol (DAG) which contributes to BK-induced hypersensitivity. DAG subsequently activates calcium-independent protein kinase $C_{\varepsilon}\left(P K C_{\varepsilon}\right)$ with resultant phosphorylation and enhanced thermal sensitivity of TRPV1 channels.

$\mathrm{IP}_{3}$, on the other hand, is involved with acute $\mathrm{BK}$-induced pain provocation, but the precise mechanisms by which this occurrs have been uncertain. Recently Liu et al (5) have shed light on the molecular mechanisms which appear to underlie BK-induced pain. Liu et al (5) appear to have uncovered 2 events which largely explain the major mechanisms of acute nociceptive signaling induced by bradykinin. $\mathrm{IP}_{3}$ releases calcium ions from the endoplasmic reticulum resulting in a rise in intracellular calcium concentration. This rise in calcium results in 2 simultaneous events in sensory neurons (5). First, the increased intracellular calcium inhibits a potassium current carried by Kv 7.2/Kv 7.3 channels known as "the M current," leading to cell depolarization. Secondly, Liu and colleagues (5) report that the rise in calcium also activates a calcium-dependent chloride current (CaCC).

When applied exogenously to a human skin blister or injected into arteries supplying viscera in laboratory animals (2), BK induces 2 major effects: (a) severe spontaneous pain and (b) increased sensitivity to painful (hyperalgesia) and nonpainful (allodynia) thermal and mechanical stimuli $(3,6)$. The findings of Liu and colleagues in a series of experiments indicate that the major effects of BK on the excitability of nociceptors are mediated by PLC- and Ca2+-dependent inhibition of M-type $\mathrm{K}+$ channels and by simultaneous opening of $\mathrm{Ca}^{2+-a c t i v a t e d} \mathrm{Cl}$ - channels (CaCCs) encoded by transmembrane protein 16A (Tmem16a). Both these effects summate to contribute to membrane depolarization in primary sensory afferents, resulting in the generation of ascending nociceptive signals (5). In small nociceptive neurons from rat dorsal root ganglia, bradykinin binds to bradykinin 2 receptors $\left(B_{2}\right)$ which couple to $G_{q / 11}$ and activate PLC (6). PLC hydrolyzes the plasma membrane phospholipid phosphatidylinositol 4,5-bisphosphate $\left(\mathrm{PIP}_{2}\right)$ and releases inositol 1,4,5-trisphosphate (IP3) into the

From: Albany Medical College, Albany, NY.

Dr. Smith is Editor-in-Chief of Pain Physician, Associate Professor \& Academic Director of Pain Management, Albany Medical College, Albany, New York.

E-mail: smithh@mail.amc.edu

Disclaimer: There was no external funding in the preparation of this manuscript. Conflict of interest: None. 
cytosol; the latter can then trigger exit of $\mathrm{Ca}^{2+}$ from $\mathrm{IP}_{3}$-sensitive intracellular stores. Both $\mathrm{PIP}_{2}$ depletion and $\mathrm{Ca}^{2+}$ release are powerful signaling events capable of regulating neuronal ion channels.

\section{M-Current}

In a series of patch-clamp experiments, BK was demonstrated to lead to inhibition of $M$ current which was always accompanied by the simultaneous activation of an inward current at $-60 \mathrm{mV}$ (5). In efforts to investigate whether "M channel" inhibition or CaCC activation contributes to BK-induced animal nocipceptive behaviors, Liu and colleagues preinjected the "M channel" opener retigabine or different $\mathrm{CaCC}$ blockers into the same plantar region of the hind paw where 5 minutes later BK was injected (5). They tested whether the "M channel" opener retigabine can reduce BK-induced nocifensive behavior, thinking that "M channels" inhibited by BK might be "rescued" with retigabine. Retigabine ( $5 \mathrm{nmol} / \mathrm{site}$ ) was able to significantly attenuate BK-induced nociceptive responses (5). In patch-clamp experiments, application of retigabine effectively reversed the inhibitory effect of BK on M current at -60 $\mathrm{mV}(5)$.

\section{Calcium-Dependent Chloride Current}

Another important mechanism that contributes to BK-induced acute nociceptive signaling involves the activation of calcium-dependent chloride channels encoded by transmembrane protein 16A. The transmembrane protein 16 (TMEM16 or anoctamin) protein family has been identified as a CaCC subunit (7-9). The gene encoding an essential CaCC subunit (Tmem16A- also know as Ano 1) has recently been identified (7-9).

Lowering intracellular $\mathrm{Cl}-\left([\mathrm{Cl}-]_{\mathrm{i}}\right)$ by using a potassium acetate-based pipette solution almost completely abolished BK-induced inward current. Furthermore, the broad-spectrum chloride channel blocker 4,4'-diisothiocyanatostilbene- 2, 2'-disulphonic acid (DIDS, $100 \mu \mathrm{M}$ ) also abolished BK-induced inward current. Other $\mathrm{Cl}^{-}$ channel blockers, niflumic acid (NFA, $100 \mu \mathrm{M}$ ) and 5nitro-2-(3 phenylpropylamino) benzoic acid (NPPB, 100 $\mu \mathrm{M})$, also effectively blocked BK-induced inward currents. The above results suggest that the inward current evoked by BK was almost exclusively due to chlo- ride channel opening (5). The B2R antagonist Hoe-140 and PLC blocker edelfosine both prevented the inward current responses, suggesting that this inward current is conducted by $\mathrm{CaCC}$ activated via the $\mathrm{PLC} / \mathrm{IP}_{3} / \mathrm{Ca}^{2+}$ pathway (5). Liu and colleagues used siRNA against Tmem16a to test whether it is a molecular correlate of the BK-induced $\mathrm{Cl}^{-}$current in small DRG neurons. Transfection of siRNA significantly reduced levels of Tmem16a mRNA and protein. Liu et al (5) also tested whether pharmacological inhibition of $\mathrm{CaCC}$ would lead to anti-nociceptive effects. Preinjection of the $\mathrm{Cl}^{-}$channel blockers DIDS (50 nmol/site) and NPPB (10 nmol/site) significantly attenuated the BK-induced nociceptive behaviors (5).

\section{SUMMARY}

Thus, it appears that the acute pain which bradykinin can induce, is dependent on both the modulation of transmembrane protein 16 leading to opening of calcium dependent chloride channels with subsequent escape of negatively charged chloride ions resulting in a less negative intracellular environment (depolarization), as well as, the closing of M-type potassium channels which stops the escape of positively charged potassium ions resulting in a more positive intracellular environment (depolarization) from intracellular potassium accumulation (Fig. 1). These 2 processes both result in depolarization of sensory neurons that may facilitate nociception.

These two mechanisms each represent a potential for antinociceptive strategies aimed at: 1.) closing TMEM16A calcium-dependent chloride channels, and/ or 2.) opening of M-type potassium channels. Retigabine and agents like it may provide analgesia by the second of these mechanisms. Retigabine's mechanism of action involves opening of neuronal Kv 7.2-7.5 voltage activated $\mathrm{K}^{+}$channels responsible for generation of the M-current. Additionally, retigabine has also been shown to increase synthesis of gamma amino butyric acid (GABA) in rat hippocampal neurons and to allosterically potentiate GABA-induced chloride currents in cultured rat cortical neurons. Behavioral studies have shown retigabine can diminish pain behaviors involving animal models of neuropathic pain (10-13). 


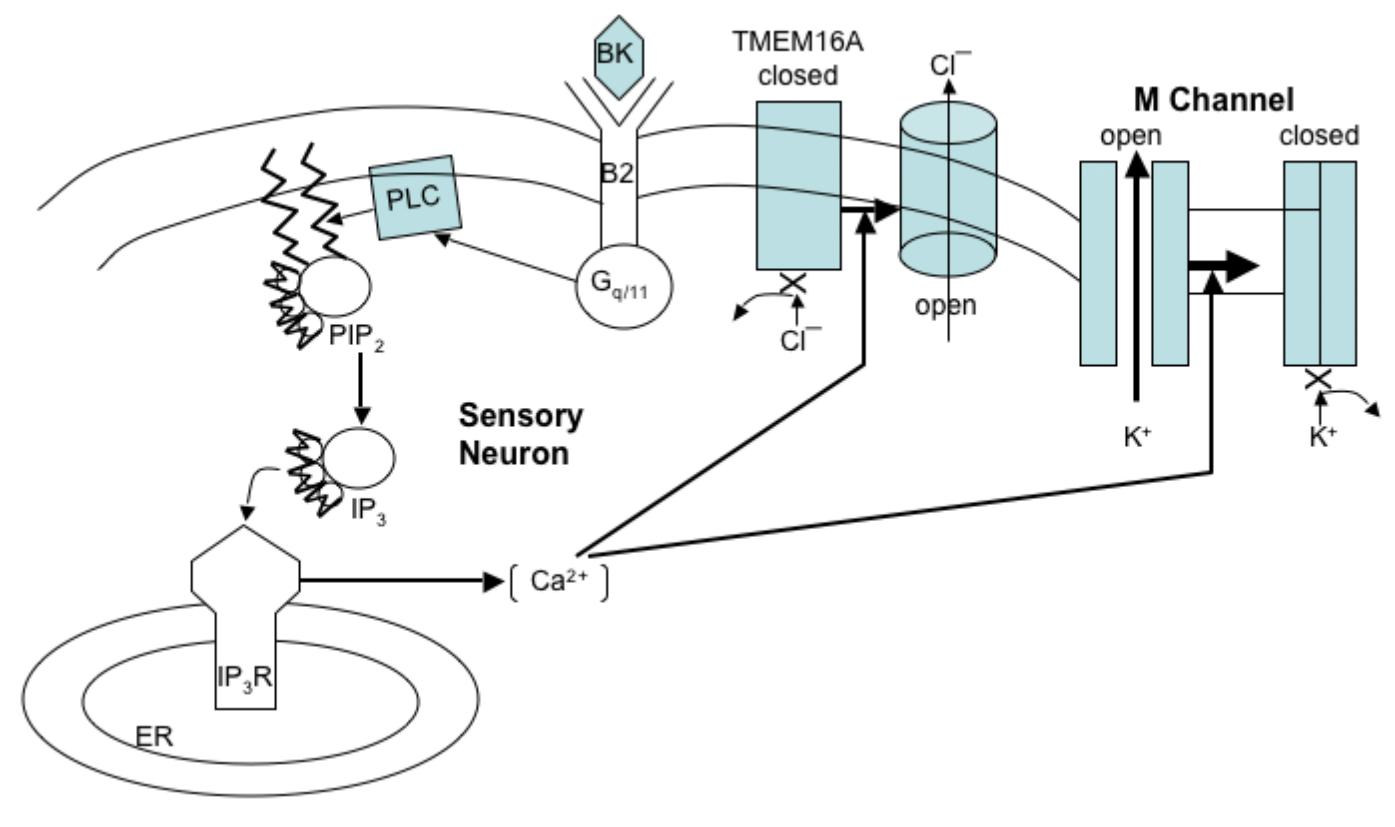

Fig. 1. Schematic of mechanisms of bradykinn-induced depolarization of sensory neurons that may facilitate nociception.

\section{References}

1. McMahon SB, Bennet DLH, Bevan S. Inflammatory mediators and modulators. In McMahon SB, Koltzenburg M (eds). Wall and Melzack's Textbook of Pain. Elsevier Churchill Livingstone, Edinburgh, United Kingdom, 2006, pp 4972.

2. Keele CA. The chemistry of pain production. Proc R Soc Med 1967; 60:419422.

3. Dray A, Perkins M. Bradykinin and inflammatory pain. Trends Neurosci 1993; 16:99-104.

4. Moreau ME, Garbacki N, Molinaro G, Brown NJ, Marceau F, Adam A. The kallikrein-kinin system: current and future pharmacological targets. I Pharmacol Sci 2005; 90:6-38.

5. Liu B, Linley JE, Du X, Zhang X, Ooi, L, Zhang H, Gamper N. The acute nociceptive signals induced by bradykinin in rat sensory neurons are mediated by inhibition of M-type $\mathrm{K}+$ channels and activation of $\mathrm{Ca} 2+-$ activated $\mathrm{Cl}$ - channels. J Clin Invest 2010;120:1240-1252.

6. Couture R, Harrisson M, Vianna RM, Cloutier F. Kinin receptors in pain and inflammation. Eur / Pharmacol 2001; 429:161-176.

7. Caputo A, Caci E, Ferrera L, Pedemonte N, Barsanti C, Sondo E, Pfeffer U, Ravazzolo R, Zegarra-Moran O, Galietta LJ. TMEM16A, a membrane protein associated with calcium-dependent chloride channel activity. Science 2008; 322:590-594.

8. Schroeder BC, Cheng T, Jan YN, Jan LY. Expression cloning of TMEM16A as a calcium-activated chloride channel subunit. Cell 2008; 134:1019-1029.

9. Yang YD, Cho H, Koo JY, Tak MH, Cho Y, Shim WS, Park SP, Lee J, Lee B, Kim BM, Raouf R, Shin YK, Oh U. TMEM16A confers receptor-activated calcium-dependent chloride conductance. Nature 2008; 455:1210-1215.

10. Wickenden A, Weifeng Y, Zou A. Retigabine, a novel anti-covulsant, enhances activation of $K C \mathrm{NQ}_{2} / \mathrm{Q}_{3}$ potassium channels. Mol Pharmacol 2000; 58:591-600.

11. Wuttke T, Seebohm G, Bail S. The new anticonvulsant Retigabine favors voltage-dependent opening of Kv7.2 (KCNQ2) channel by binding to its activation gate. Mol Pharmacol 2005; 67:1009-1017.

12. Marrion NV. Control of M-current. Annu Rev Phsyciol 1997; 59:483-504.

13. Blackburn-Munro G, Jensen BS. The anticonvulsant retigabine attenuates nociceptive behaviours in rat models of persistent and neuropathic pain. Eur J Pharmacol 2003; 460:109-116. 
